\title{
OPTICALLY INJECTED SEMICONDUCTOR LASER FOR PHOTONIC MICROWAVE FREQUENCY MIXING IN RADIO-OVER-FIBER
}

\author{
X. Fu, C. Cui, and S.-C. Chan \\ Department of Electronic Engineering \\ City University of Hong Kong \\ Hong Kong, China
}

\begin{abstract}
Nonlinear dynamics of an optically injected semiconductor laser for microwave frequency mixing are experimentally investigated. Through optical injection, the laser is driven into the nonlinear periodone (P1) dynamical state and acts as a broadly tunable photonic microwave oscillator. With an external current modulation at an intermediate frequency, the $\mathrm{P} 1$ oscillation is modulated accordingly, thus achieving frequency up-conversion beyond the laser bandwidth. Using a 2.5-Gbps-grade laser, binary phase-shift keying (BPSK) data signal is up-converted from an intermediate frequency of $2 \mathrm{GHz}$ to a subcarrier frequency as high as $20 \mathrm{GHz}$. While the direct modulation bandwidth limits the intermediate frequency, it does not limit the subcarrier frequency. The approach uses only low-cost lasers and requires no high-speed electronic mixers.
\end{abstract}

\section{INTRODUCTION}

Nonlinear dynamics of semiconductor lasers invoked by optical injection have been investigated for various applications in the last decade [1-5]. Among the applications is the radio-over-fiber (RoF) system in which radio or wireless signals on a microwave subcarrier are transmitted through an optical carrier over optical fibers. Sharing the intrinsic merits of optical fibers, RoF systems have the advantages such as reduced size, weight, and cost, low attenuation, immunity to electromagnetic interference, low dispersion, and high data transfer capacity $[6,7]$. The original data to be transmitted from the central office is generally at the baseband or an intermediate frequency. It has to be up-converted to the microwave subcarrier frequency for downlink

Corresponding author: S.-C. Chan (scchan@cityu.edu.hk). 
transmission. In order to reduce the cost on high-speed electronics, it is desired that microwave generation and frequency up-conversion are performed optically at the central office.

Various approaches of such optical microwave frequency mixing have been investigated. The nonlinearities of a semiconductor optical amplifier (SOA) [8], a highly nonlinear fiber (HNLF) [9], and a base station electronic mixer [10] were considered. However, all these methods required high-speed external modulators that are generally expensive and lossy. As an alternative, a technique based on modulating a mode-locked multisection laser was reported [11]. The approach employed no external modulators, but the tunability of the subcarrier frequency was inherently limited by the laser structure, which required sophisticated cavity design. Recently, the nonlinear response of a vertical-cavity surface-emitting laser (VCSEL) biased close to threshold was utilized [12]. While the approach was relatively simple, the subcarrier frequency was limited by the intrinsic direct modulation bandwidth to only $3.1 \mathrm{GHz}$.

The nonlinear dynamical period-one (P1) oscillation of optically injected semiconductor lasers offers a solution [1]. Through optical injection of proper power and detuning frequency, P1 oscillation can be invoked through undamping the relaxation oscillation. Photonic microwave is generated even without direct modulation of the bias current. The bandwidth limitation imposed by the direct modulation response can be circumvented, where tunable generation of microwave frequencies six-fold beyond the relaxation oscillation frequency has been investigated [3]. For example, stable subcarrier frequency of $40 \mathrm{GHz}$ has been experimentally obtained [13]. The P1 oscillation has been investigated for both RoF downlink and uplink data transmissions $[2,5]$. However, these methods required first electrically mixing data to the subcarrier frequency before modulating the lasers. To the best of our knowledge, incorporation of frequency up-conversion using the P1 oscillation for RoF applications has not been investigated.

In this Letter, an ordinary 2.5-Gbps-grade 1.55- $\mu \mathrm{m}$ single-mode distributed feedback (DFB) laser is applied for all-optical microwave frequency up-conversion. Under proper optical injection, the laser is forced into P1 oscillation and experiences intensity oscillation at a characteristic frequency $f_{0}$, which is tuned to $18 \mathrm{GHz}$ for illustration. A data stream in binary phase-shift keying (BPSK) at an intermediate frequency $f_{\mathrm{IF}}=2 \mathrm{GHz}$ then modulates the laser directly. The P1 oscillation at $f_{0}$ is modulated by $f_{\mathrm{IF}}$ accordingly. Data is up-converted to both the upper sideband (USB) at $f_{0}+f_{\mathrm{IF}}=20 \mathrm{GHz}$ and the lower sideband (LSB) at $f_{0}-f_{\mathrm{IF}}=16 \mathrm{GHz}$. Although the subcarriers $f_{0} \pm f_{\mathrm{IF}}$ are already significantly greater than the original laser bandwidth, they 
can be easily increased by increasing the optical injection strength or detuning frequency. The construction of the central office uses no highspeed electronic mixers. Up-conversion to the subcarrier frequency is performed by the laser without being limited by the direct modulation bandwidth.

The experimental approach in this work is demonstrated for the first time and is consistent with previous numerical simulations, which were based on the well established rate-equation model of optically injected semiconductor lasers [14].

\section{EXPERIMENTAL SETUP}

\subsection{Central Office}

The schematic of the experimental setup is shown in Fig. 1. Both the master laser and the slave laser are single-mode DFB lasers of the same model (Mitsubishi ML920T43S-01). The lasers emit at around $1.55 \mu \mathrm{m}$ and have been designed with electrical bandwidth supporting only up to $2.5 \mathrm{Gbps}$. At the central office, light from the master laser is injected into the slave laser. The combination of the half-wave plates, the Faraday rotator, and the polarizing beam splitter forms an optical

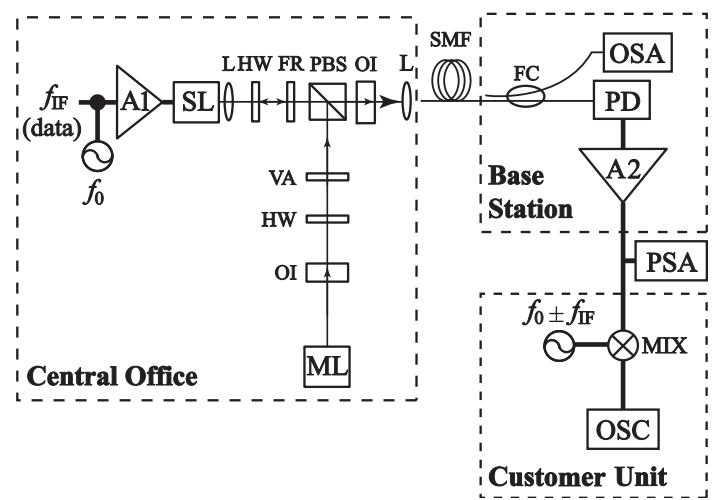

Figure 1. Schematic of the optical injection system for mixing $f_{0}$ and $f_{\mathrm{IF}}$ for an RoF downlink. ML: Master laser; SL: Slave laser; L: Lens; HW: Half-wave plate; FR: Faraday rotator; PBS: Polarizing beam splitter; VA: Variable attenuator; CIR: Optical circulator; OI: Optical isolator; SMF: Single-mode fiber; FC: Fiber coupler; PD: Photodetector; A1, A2: Electrical amplifiers; PSA: Power spectrum analyzer; OSA: Optical spectrum analyzer. MIX: Electronic mixer; OSC: Oscilloscope. Thin lines: Optical paths. Thick lines: Electrical cables. 
circulator which ensures unidirectional optical injection. While the master laser can be any tunable single-mode laser, our experiment employed the laser of the same model as the slave laser to ensure the wavelengths are nearly matched. Nonetheless, fabrication variation of the lasers causes a slight difference in their wavelengths. Thus, both lasers are temperature stabilized so that their free-running wavelengths can be temperature-tuned.

Different dynamics can be invoked in the slave laser through optical injection by varying the injection conditions, including the injection power $P_{\mathrm{i}}$ and the injection detuning frequency $f_{\mathrm{i}}$, which is defined as the optical frequency difference between the master laser and the free-running slave laser. When the injection forces the slave laser into the nonlinear P1 dynamical oscillation, its output intensity oscillates at a microwave frequency $f_{0}$, even without any current modulation [1]. Tuning of $f_{0}$ is achieved through varying $P_{\mathrm{i}}$ and $f_{\mathrm{i}}$. The injection power $P_{\mathrm{i}}$ is controlled at the variable attenuator, while the injection detuning frequency $f_{\mathrm{i}}$ is controlled by the bias current and the temperatures of the lasers.

\subsection{Base Station}

The output of the slave laser is transmitted over a 4-km optical fiber to the base station. The optical signal is separated into two branches. One is monitored by an optical spectrum analyzer (HP 86142A). The other branch is detected by a photodetector (Newport AD-10ir), amplified by $40 \mathrm{~dB}$ electrically using two microwave amplifiers (HP 83006A and HP 83017A), and monitored by a power spectrum analyzer (Agilent N9010A). As an option, a current modulation at $f_{0}$ can be applied to the slave laser to stabilize the P1 oscillation frequency [1], but it is not essential for the frequency mixing described below.

In order to perform all-optical microwave frequency mixing, a current modulation at an intermediate frequency $f_{\mathrm{IF}}$ is applied to the slave laser through a 20-dB amplifier (HP 83006A). The P1 oscillation at $f_{0}$ is modulated accordingly, generating mixing products at $f_{0} \pm f_{\mathrm{IF}}$ as subcarrier frequencies for RoF. When $f_{\mathrm{IF}}$ carries data from a pattern generator (Agilent N4906B), the data is up-converted to the subcarriers for transmission to the customer unit.

\subsection{Customer Unit}

The customer unit receives the downlink signal at the subcarriers $f_{0} \pm f_{\mathrm{IF}}$. For illustration, our experiment simply uses a local oscillator (HP $83630 \mathrm{~B})$ at $f_{0}+f_{\mathrm{IF}}$ or $f_{0}-f_{\mathrm{IF}}$ to electrically mix with the downlink signal. The local oscillator gives $18-\mathrm{dBm}$ and the conversion loss is 
$8 \mathrm{~dB}$. The demodulated data is sent to a real-time digital oscilloscope (DSO90254A) of $200 \mathrm{MHz}$ effective bandwidth for observation.

\section{EXPERIMENTAL RESULTS}

\subsection{P1 Oscillation}

The master laser is biased at $133.9 \mathrm{~mA}$ and temperature stabilized at $18.00^{\circ} \mathrm{C}$; while the slave laser is biased at $40.0 \mathrm{~mA}$ and temperature stabilized at $27.00^{\circ} \mathrm{C}$. The threshold of the slave laser is $8.0 \mathrm{~mA}$. Under this operating condition, the slave laser emits $8.17 \mathrm{~mW}$ when it is not under optical injection. The master laser is positively detuned from the slave laser with an injection detuning frequency of $f_{\mathrm{i}}=5 \mathrm{GHz}$. When the injection power is adjusted to $P_{\mathrm{i}}=5.25 \mathrm{~mW}, \mathrm{P} 1$ oscillation at $f_{0}=18 \mathrm{GHz}$ is generated. Fig. 2 shows the optical spectrum centered at the free-running frequency of the slave laser at $193.745 \mathrm{THz}$. Due to optical injection, a regenerative component at $f_{\mathrm{i}}$ above the free-running frequency is observed, as indicated by the arrow. The nonlinear dynamical P1 oscillation generates the sidebands equally separated by $f_{0}$. A beat signal is thus obtained as a peak at $f_{0}$ in the power spectrum in Fig. 3(a). The microwave oscillation is obtained through the nonlinear dynamics alone without applying any current modulation. By fixing the injection detuning frequency at $f_{\mathrm{i}}=5 \mathrm{GHz}$, the generated microwave can be tuned from $12 \mathrm{GHz}$ to $21 \mathrm{GHz}$ by varying the injection power $P_{\mathrm{i}}$ from $1.11 \mathrm{~mW}$ to $9.37 \mathrm{~mW}$, as Fig. 3(b) shows. The $\mathrm{P} 1$ oscillation frequency $f_{0}$ increases nearly linearly with $\sqrt{P_{\mathrm{i}}}[15]$.

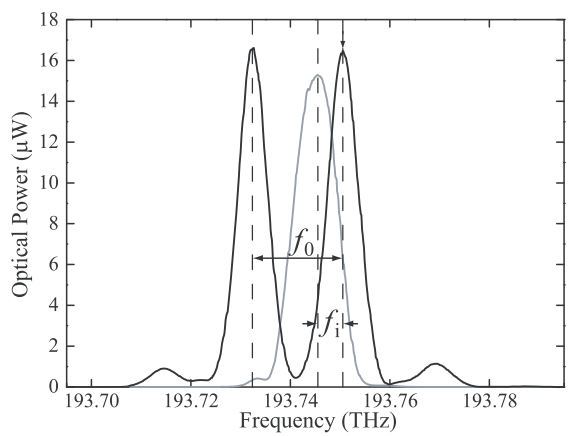

Figure 2. Optical spectrum of the emission from the slave laser under no current modulation. The gray curve is obtained without optical injection. The black curve is obtained with optical injection of $f_{\mathrm{i}}=5 \mathrm{GHz}$ and $P_{\mathrm{i}}=5.25 \mathrm{~mW}$. The slave laser exhibits $\mathrm{P} 1$ oscillation of $f_{0}=18 \mathrm{GHz}$. (Resolution bandwidth $=7.5 \mathrm{GHz}$ ). 


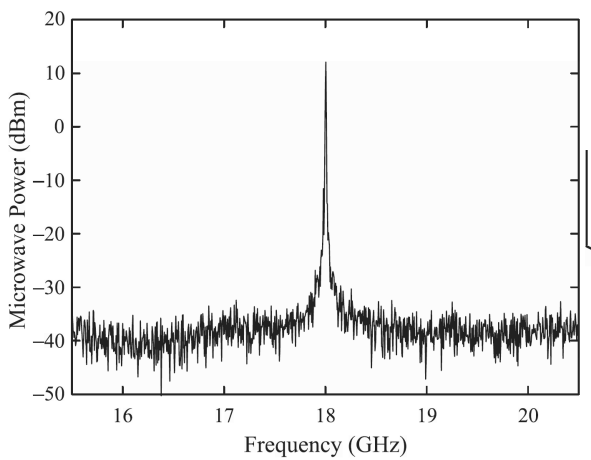

(a)

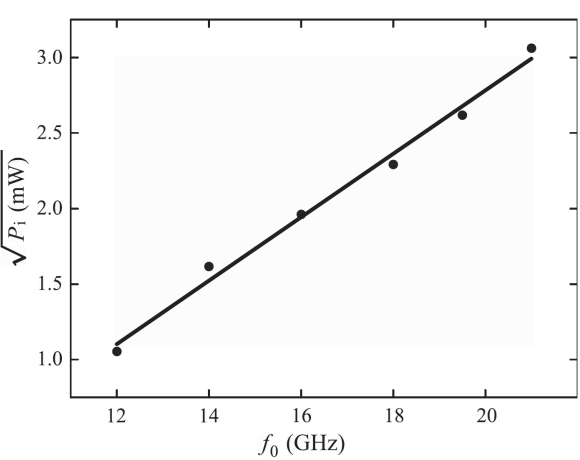

(b)

Figure 3. (a) Power spectrum of the slave laser under P1 oscillation at $f_{0}=18 \mathrm{GHz}$. No current modulation is applied. (b) Tunability of $f_{0}$ by varying $P_{\mathrm{i}}$ under constant $f_{\mathrm{i}}=5 \mathrm{GHz}$.

It is worth noting that, due to imperfections in optical alignment, only a small portion of the optical injection power actually couples into the slave laser. While it is difficult to directly measure the actual injection power ratio, the experimental results in Fig. 3(b) can be compared to numerical simulation results to deduce the injection power ratio. Using the well established rate equations of intracavity optical field and charge carrier density, together with the experimentally obtained laser dynamical parameters, the $\mathrm{P} 1$ oscillation frequency $f_{0}$ can be obtained as a function of the injection power ratio [3]. From such approach, the injection power ratio is estimated to be about $1 \%$ for Fig. 3(a). The ratio is small because of the experimental challenges in injecting light into the output facets of the slave laser, which typically has only a few micrometers in height and width.

In Fig. 3, while spontaneous emission noise and intrinsic fluctuation of the system lead to a microwave linewidth on the order of $10 \mathrm{MHz}$, it can be narrowed by a weak current modulation of $5 \mathrm{dBm}$ at $f_{0}$ [1]. The modulation remains on for the following characterization of frequency mixing, up-conversion bandwidth, up-conversion power, and data transmission.

\subsection{Frequency Mixing}

For frequency up-conversion, $f_{0}$ is kept at $18 \mathrm{GHz}$ and an additional current modulation at the intermediate frequency $f_{\mathrm{IF}}=2 \mathrm{GHz}$ and power $P_{\mathrm{IF}}=-4 \mathrm{dBm}$ is applied to the slave laser. Fig. 4 shows that the $\mathrm{P} 1$ oscillation is modulated accordingly. Up-converted signals at both $f_{0}+f_{\mathrm{IF}}=20 \mathrm{GHz}$ and $f_{0}-f_{\mathrm{IF}}=16 \mathrm{GHz}$ are clearly identified. 
While the USB is slightly stronger than the LSB in the present P1 state, the LSB can become stronger than the USB when a different P1 state is used.

The up-conversion bandwidth of the photonic microwave mixer is characterized by varying $f_{\mathrm{IF}}$, where the optical injection conditions remain constant and $P_{\mathrm{IF}}$ is kept constant at $-4 \mathrm{dBm}$. Fig. 5(a) shows the microwave powers at the USB and the LSB as solid and

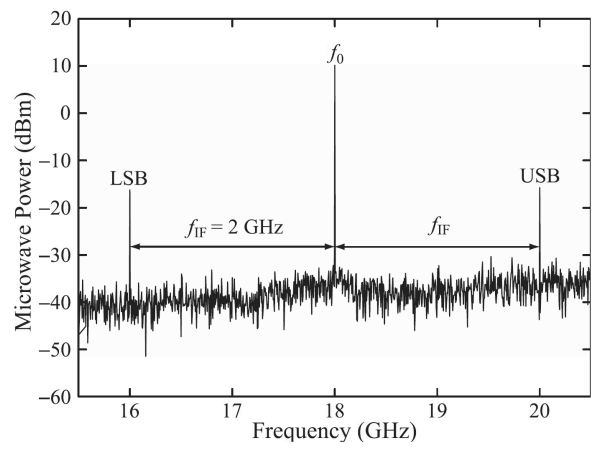

Figure 4. Power spectrum of the slave laser under P1 oscillation at $f_{0}=18 \mathrm{GHz}$ and current modulation at $f_{\mathrm{IF}}=2 \mathrm{GHz}$. The input current modulation power is $P_{\mathrm{IF}}=-4 \mathrm{dBm}$. Freuqency up-conversion from $f_{\mathrm{IF}}$ to $f_{0} \pm f_{\mathrm{IF}}$ is observed.

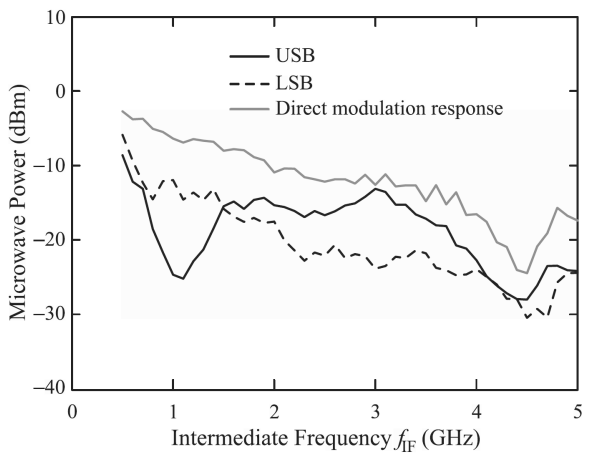

(a)

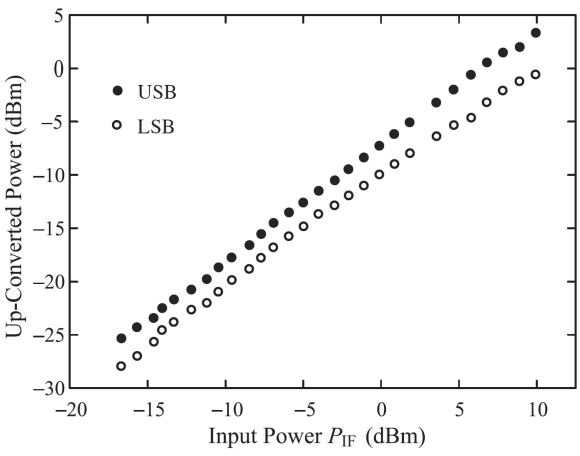

(b)

Figure 5. (a) Up-conversion power from the photonic microwave mixing of $f_{\mathrm{IF}}$ and $f_{0}=18 \mathrm{GHz}$. The solid and dashed dark curves correspond to the USB and the LSB, respectively. For comparison, the gray curve is the direct modulation response of the slave laser. Modulation power is kept at $P_{\mathrm{IF}}=-4 \mathrm{dBm}$. (b) The USB and LSB power versus $P_{\mathrm{IF}}$ at $f_{\mathrm{IF}}=2 \mathrm{GHz}$. 
dashed dark curves, respectively. The gray curve shows the direct modulation response of the slave laser for comparison. The upconversion responses roughly follow the trend of the direct modulation response. This indicates that the intermediate frequency $f_{\mathrm{IF}}$ is limited by the modulation bandwidth of the slave laser, even though the P1 frequency $f_{0}$ is not.

Due to the antiguidance effect associated with a positive linewidth enhancement factor, the optical spectrum of P1 oscillation is generally asymmetric [16-18]. Likewise, asymmetry in the power levels of the USB and LSB is always observed in the mixing experiments. Details require thorough numerical simulation and is beyond the current scope. Nevertheless, such asymmetry was also observed in a related simulation study [14]. Furthermore, as shown in the inset of Fig. 5, the signal powers at both subcarriers increase approximately linearly with $P_{\mathrm{IF}}$. For a noise floor of $-30 \mathrm{dBm}$, there is a linear operational range of more than $20 \mathrm{~dB}$ for both the USB and the LSB. Both sidebands are applied as the subcarriers in the following demonstration of data upconversion.

\subsection{Data Up-Conversion}

The proposed photonic microwave mixer is further explored for data up-conversion. When BPSK data is modulated on $f_{\mathrm{IF}}$, it is upconverted to the subcarrier frequencies $f_{0} \pm f_{\mathrm{IF}}$ accordingly. The power spectrum with up-converted 155 -Mbps data using $f_{0}=18 \mathrm{GHz}$ and $f_{\mathrm{IF}}=2 \mathrm{GHz}$ is shown in Fig. 6(a). Data sidebands are clearly

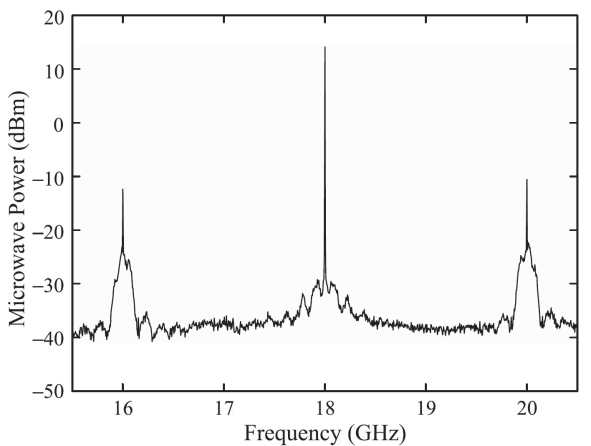

(a)

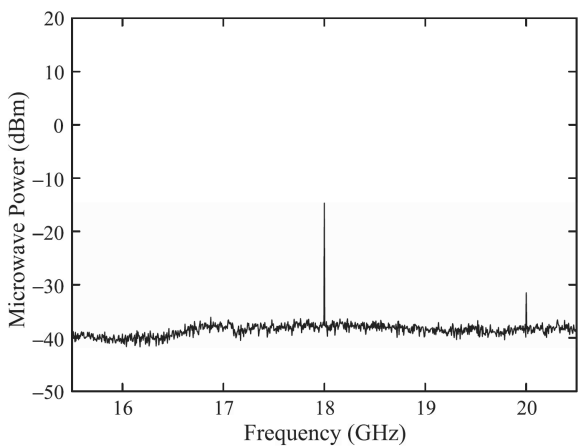

(b)

Figure 6. Power spectrum of the slave laser under current modulation at $f_{0}=18 \mathrm{GHz}$ and $f_{\mathrm{IF}}=2 \mathrm{GHz}$. The modulation at $f_{\mathrm{IF}}$ carries BPSK data at $155 \mathrm{Mbps}$. (a) Up-converted signal when optical injection is applied. (b) Up-converted signal when optical injection is removed. 


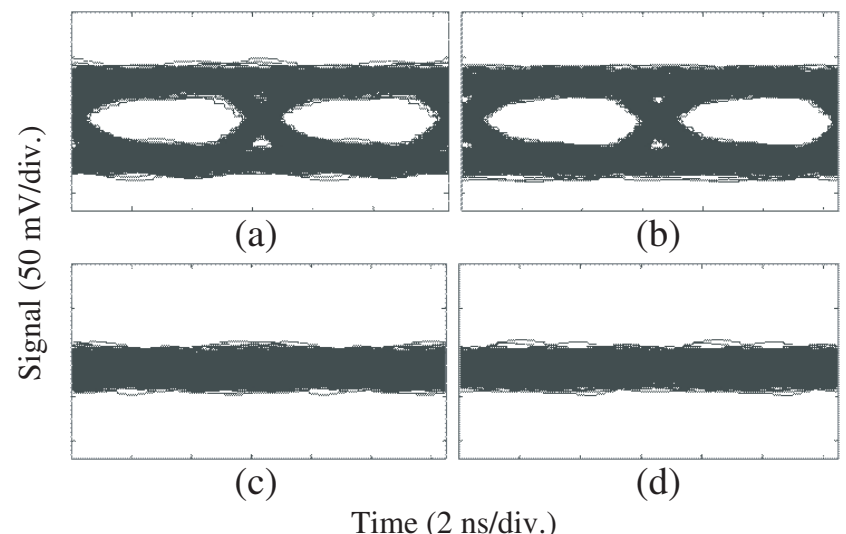

Figure 7. Eye diagrams of the data recovered at (a) 16-GHz LSB with optical injection. (b) 20-GHz USB with optical injection. (c) 16$\mathrm{GHz}$ LSB without optical injection. (d) 20-GHz USB without optical injection.

identified at both the USB and the LSB. The up-converted signals can be radiated to wireless customer units in real applications, although they are sent directly to the customer unit here for illustration. The customer unit directly demodulates the signals by electrically mixing them with a local oscillator at $f_{0} \pm f_{\mathrm{IF}}$. Data is recovered with biterror rates (BERs) of $8.02 \times 10^{-11}$ at the USB and $8.11 \times 10^{-10}$ at the LSB. The corresponding eye diagrams are shown in Figs. 7(a) and (b). By contrast, no evidence of data up-conversion is identified in the power spectrum in Fig. 6(b) when no optical injection is applied. Any effort to realize data recovery is in vain as the corresponding eye diagrams are completely closed, as shown in Figs. 7(c) and (d). Therefore, optical injection is essential to generating the P1 oscillation as well as performing frequency mixing.

In order to show the system tunability, measurements are made at $\left(f_{0}, f_{\mathrm{IF}}\right)=(18 \mathrm{GHz}, 2 \mathrm{GHz}),(18 \mathrm{GHz}, 2.3 \mathrm{GHz})$, and $(16 \mathrm{GHz}, 2 \mathrm{GHz})$, where $f_{0}$ is tuned by varying the injection conditions. The quality of data up-conversion is quantified by finding the BER. When there is no optical injection, the BER remains on the order of $10^{-1}$ as no effective data up-conversion is realized. For the proposed system with optical injection, the BER decreases as the received optical power increases, as Fig. 8 shows. For $\left(f_{0}, f_{\mathrm{IF}}\right)=(18 \mathrm{GHz}, 2 \mathrm{GHz})$, it is observed that the USB has a lower BER than the LSB. This is expected from Fig. 4 as the power of USB is stronger. At a received optical power level of around $-2.44 \mathrm{dBm}$, data up-conversion with BER of less than $10^{-9}$ can always be achieved for all the settings in Fig. 8. 


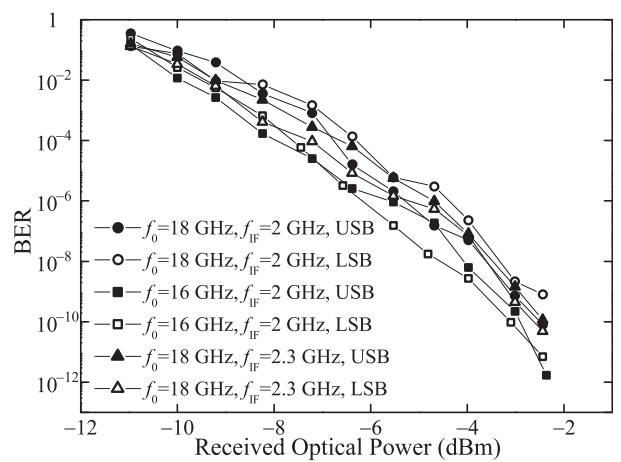

Figure 8. BER of the system under different P1 oscillation frequencies $f_{0}$ and intermediate frequencies $f_{\mathrm{IF}}$. Data rate $=155 \mathrm{Mbps}$.

\section{CONCLUSION}

An optically injected semiconductor laser in the P1 oscillation state is applied for photonic microwave frequency mixing. The direct modulation bandwidth limits only the intermediate frequency, but not the subcarrier frequency. With an ordinary 2.5-Gbps-grade laser, RoF downlink BPSK signals are up-converted from $2 \mathrm{GHz}$ to a subcarrier frequency of $20 \mathrm{GHz}$. The system at the central office requires no highspeed lasers, electronic mixers, or external modulators.

\section{ACKNOWLEDGMENT}

The work described in this paper was fully supported by a grant from City University of Hong Kong (Project No. 7008046) and a grant from the Research Grants Council of Hong Kong, China (Project No. CityU 111308).

\section{REFERENCES}

1. Simpson, T. B. and F. Doft, "Double-locked laser diode for microwave photonics applications," IEEE Photon. Technol. Lett., Vol. 11, 1476, 1999.

2. Kaszubowska, A., P. Anandarajah, and L. P. Barry, "Improved performance of a hybrid radio/fiber system using a directly modulated laser transmitter with external injection," IEEE Photon. Technol. Lett., Vol. 14, 233, 2002.

3. Chan, S. C., S. K. Hwang, and J. M. Liu, "Period-one oscillation 
for photonic microwave transmission using an optically injected semiconductor laser," Opt. Express, Vol. 15, 14921, 2007.

4. Hwang, S. K., H. F. Chen, and C. Y. Lin, "All-optical frequency conversion using nonlinear dynamics of semiconductor lasers," Opt. Lett., Vol. 34, 812, 2009.

5. Cui, C., X. Fu, and S. C. Chan, "Double-locked semiconductor laser for radio-over-fiber uplink transmission," Opt. Lett., Vol. 34, 3821, 2009.

6. Shahoei, H., H. Ghafoori-Fard, and A. Rostami, "A novel design methodology of multi-clad single mode optical fiber for broadband optical networks," Progress In Electromagnetics Research, PIER 80, 253-275, 2008.

7. Ying, C. L., H.-H. Lu, W.-S. Tsai, H.-C. Peng, and C.-H. Lee, "To employ SOA-based optical SSB modulation technique in fullduplex RoF transport systems," Progress In Electromagnetics Research Letters, Vol. 7, 1-13, 2009.

8. Yang, W., M. Zhang, H. Han, L. Cai, and P. Ye, "Analysis of photonic frequency up-conversion for radio-on-fiber applications using two-pump four-wave mixing in semiconductor optical amplifiers," Opt. Eng., Vol. 47, 075044, 2008.

9. Gao, Y., S. Gao, and H. Ou, "All-optical frequency converter based on fiber four-wave mixing for bidirectional radio-over-fiber systems," Microwave Opt. Technol. Lett., Vol. 51, 1542, 2009.

10. Li, J., T. Ning, L. Pei, and C. Qi, "Millimeter-wave radio-overfiber system based on two-step heterodyne technique," Opt. Lett., Vol. 34, 3136, 2009.

11. Lee, K. H., W. Y. Choi, Y. A. Leem, and K. H. Park, "Harmonic millimeter-wave generation and frequency up-conversion using a passively mode-locked multisection DFB laser under external optical injection," IEEE Photon. Technol. Lett., Vol. 19, 161, 2007.

12. Constant, S. B., Y. L. Guennec, G. Maury, G. Nguyen, M. Lourdiane, and B. Cabon, "Low-cost all-optical up-conversion of digital radio signals using a directly modulated 1550-nm VCSEL," IEEE Photon. Technol. Lett., Vol. 20, 120, 2008.

13. Chan, S. C., S. K. Hwang, and J. M. Liu, "Radio-over-fiber transmission from an optically injected semiconductor laser in period-one state," Proc. of SPIE, Vol. 6468, 646811, 2007.

14. Fu, X., C. Cui, and S. C. Chan, "A photonic microwave mixer using an optically injected semiconductor laser for RoF applications," OptoElectronics and Communications Conference, ThLP71, 2009. 
15. Chan, S. C. and J. M. Liu, "A photonic microwave mixer using an optically injected semiconductor laser for RoF applications," IEEE J. Select. Topics Quantum Electron., Vol. 10, 1025, 2004.

16. Simpson, T. B., J. M. Liu, A. Gavrielides, and S. D. Jaycor, "Small-signal analysis of modulation characteristics in a semiconductor laser subject to strong optical injection," IEEE J. Quantum Electron., Vol. 32, 1456, 1996.

17. Chan, S. C., "Analysis of an optically injected semiconductor laser for microwave generation," IEEE J. Quantum Electron., Vol. 45, 421, 2010.

18. Al-Hosiny, N. M., I. D. Henning, and M. J. Adams, "Correlation of electron density changes with optical frequency shifts in optically injected semiconductor lasers," IEEE J. Quantum Electron., Vol. 42, 570, 2006. 